\title{
Antibiotic-resistant Pseudomonas aeruginosa infection in patients with bronchiectasis: prevalence, risk factors and prognostic implications
}

This article was published in the following Dove Press journal: International Journal of COPD

\author{
Yong-hua Gao',* \\ Wei-jie Guan ${ }^{2, *}$ \\ Ya-nan Zhu ${ }^{3}$ \\ Rong-chang Chen ${ }^{2}$ \\ Guo-jun Zhang' \\ 'Department of Respiratory and \\ Critical Care Medicine, The First \\ Affiliated Hospital of Zhengzhou \\ University, Zhengzhou, Henan, ${ }^{2}$ State \\ Key Laboratory of Respiratory \\ Disease, National Clinical Research \\ Center for Respiratory Disease, \\ Guangzhou Institute of Respiratory \\ Diseases, The First Affiliated Hospital \\ of Guangzhou Medical University, \\ Guangzhou, Guangdong, ${ }^{3}$ Department \\ of Emergency Medicine, The First \\ Affiliated Hospital of Zhengzhou \\ University, Zhengzhou, Henan, \\ People's Republic of China \\ *These authors contributed equally \\ to this work
}

Correspondence: Guo-jun Zhang Department of Respiratory and Critical Care Medicine, The First Affiliated Hospital of Zhengzhou University, I Jianshe East Road, Zhengzhou, Henan 450052, People's Republic of China Email zhanggj1966@sina.com

Rong-chang Chen

State Key Laboratory of Respiratory Disease, National Clinical Research Center for Respiratory Disease, Guangzhou Institute of Respiratory Disease, The First Affiliated Hospital of Guangzhou Medical University, I5I Yanjiang Road, Guangzhou, Guangdong 510120, People's Republic of China

Email chenrc@vip.163.com
Background and aims: Pseudomonas aeruginosa (PA) is the most common pathogen in bronchiectasis and frequently develops resistance to multiple classes of antibiotics, but little is known about the clinical impacts of PA-resistant (PA-R) isolates on bronchiectasis. We, therefore, investigated the prevalence, risk factors and prognostic implications of PA-R isolates in hospitalized bronchiectasis patients.

Patients and methods: Between June 2011 and July 2016, data from adult bronchiectasis patients isolated with PA at the First Affiliated Hospital of Zhengzhou University were retrospectively analyzed. PA was classified as PA-R in case antibiogram demonstrated resistance on at least one occasion.

Results: Seven hundred forty-seven bronchiectasis patients were assessed. Of these, 147 (19.7\%) had PA isolate in the sputum or bronchoscopic culture. PA-R and PA-sensitive accounted for $88(59.9 \%)$ and $59(31.1 \%)$ patients, respectively. In multivariate model, factors associated with $\mathrm{PA}-\mathrm{R}$ isolate in bronchiectasis included prior exposure to antibiotics (odds ratio $[\mathrm{OR}]=6.18$ ), three or more exacerbations in the previous year $(\mathrm{OR}=2.81)$, higher modified Medical Research Council dyspnea scores $(\mathrm{OR}=1.93)$ and greater radiologic severity $(\mathrm{OR}=1.15)$. During follow-up (median: 26 months; interquartile range: 6-59 months), 36 patients died, of whom $24(66.7 \%)$ had PA-R isolate at baseline. However, PA-R isolate was not associated with greater all-cause mortality in bronchiectasis.

Conclusion: PA-R infection is common among bronchiectasis patients, mainly determined by prior exposure to antibiotics, frequent exacerbations, more pronounced dyspnea and more severe radiologic involvement. However, PA-R isolate is not an independent risk factor for all-cause mortality in bronchiectasis.

Keywords: bronchiectasis, antibiotic resistance, Pseudomonas aeruginosa, risk factors, mortality

\section{Introduction}

Bronchiectasis is a chronic suppurative lung disease characterized radiologically by permanent dilation of bronchi and is associated with a considerable morbidity and mortality. ${ }^{1}$ Patients with bronchiectasis are frequently colonized with potentially pathogenic microorganisms (PPMs), which readily trigger airway inflammation and destruction that collectively perpetuate the vicious cycle leading to disease progression., ${ }^{2,3}$ PPMs (particularly Pseudomonas aeruginosa [PA] and Haemophilus influenzae) have been dominant microorganisms isolated from sputum in bronchiectasis. ${ }^{4-6}$ PA accounts for $21.4 \%$ of cases with bacterial colonization and is associated with accelerated lung function decline, poorer quality of life, greater risk of hospitalization and exacerbations, and increased mortality. ${ }^{7}$ Accordingly, the British Thoracic Society guidelines 
highlighted the importance of prompt evaluation of PA infection and resistance patterns for implementing optimal management. ${ }^{1}$

The prevalence of PA-resistant (PA-R) infection has been increasing in patients with chronic lung diseases, such as cystic fibrosis (CF) and COPD ${ }^{8-10}$ Nevertheless, the association between PA-R infection and prognosis in $\mathrm{CF}$ and COPD remains controversial. ${ }^{10,11}$ A nationwide study in Australia ${ }^{11}$ documented progressively increasing prevalence of multidrug-resistant (MDR)-PA which was not associated with worse clinical outcomes in $\mathrm{CF}$, nor was the isolation of PA-R being linked to higher mortality in COPD. ${ }^{10}$ Currently, there are scant data regarding clinical implications of PA-R infections in bronchiectasis. To date, only one retrospective study ${ }^{12}$ documented that $\mathrm{PA}$ infection occurred regardless of lung function impairment and that $11 \%$ of patients were infected with MDR strains. We, therefore, conducted a retrospective study to determine the prevalence, risk factors and long-term clinical impact of PA-R infection in bronchiectasis.

\section{Patients and methods}

\section{Patient selection}

A retrospective cohort study was performed on 813 consecutive bronchiectasis patients hospitalized in The First Affiliated Hospital of Zhengzhou University between July 2011 and June 2016. Bronchiectasis was confirmed by International Code for Disease- 9 codes combined with chest high-resolution computed tomography (HRCT) from hospital information system. Patients without chest HRCT or aged $<16$ years, or presented as traction bronchiectasis (assessed with chest HRCT) were excluded. Patients were also excluded from the analysis if they did not have PA being isolated from a sputum or bronchoscopic culture during the study period. This research was approved by the Ethics Committee of The First Affiliated Hospital of Zhengzhou University, which waived the need for informed consent for this retrospective, non-interventional design using anonymized patient data.

\section{Clinical data retrieval}

Data on baseline characteristics (age, sex, body mass index [BMI], smoking history, etiological investigations), radiographic extension, spirometry (forced expiratory volume in 1 second $\left[\mathrm{FEV}_{1}\right] \%$ predicted and forced vital capacity $\%$ predicted), modified Medical Research Council (mMRC) dyspnea score, comorbidities and maintenance therapy were collected by chart review. Radiographic extension was assessed by the modified Reiff score based on the number of bronchiectatic lobes (with the lingula lobe being scored as a separate lobe) and the severity of bronchial dilatation (tubular: 1 point, varicose: 2 points, cystic: 3 points), with the maximum score being $18 .{ }^{13}$ Etiological investigations were assessed based on the protocol recommended by Pasteur et al. ${ }^{13} \mathrm{CF}$ and $\alpha_{1}$-antitrypsin deficiency have been scarcely reported in Asian countries, therefore routine screening was not conducted. ${ }^{14}$

\section{Microbiologic evaluation}

Spontaneous sputum or bronchoscopic samples were sampled for each eligible patient after hospitalization. Patients were instructed to expectorate sputum into sterile plastic containers following several cycles of deep cough. Sputum or bronchoscopic samples were sent for culture within 2 hours, and were deemed qualified for analysis if $>25$ leukocytes and $<10$ squamous epithelial cells were observed per lowpower field for sputum samples. The most purulent portion of sputum was separated from saliva and placed on blood, chocolate and Sabouraud agar. A cutoff for bacterial load of $10^{3}$ colony-forming units per microliter or greater was defined as positive culture. ${ }^{4}$ Bacteria were classified into PPM strains, including PA, Klebsiella pneumoniae, Escherichia coli, Acinetobacter baumannii, Enterobacter cloacae and other pathogenic microorganisms (at the investigator's discretion). The microorganism isolation and antibiotic susceptibility tests were conducted by laboratory technicians blinded to patients' clinical characteristics.

Sensitivity testing was carried out using the agar disk diffusion method according to the Clinical Laboratory Standards Institute guidelines and resistance cutoffs. ${ }^{15}$ Antimicrobial agents employed in drug susceptibility tests included levofloxacin, ciprofloxacin, amikacin, ceftazidime, colistin, gentamicin, meropenem, piperacillin-tazobactam, ticarcillin-clavulanic acid and tobramycin. The minimum inhibitory concentration interpretive standards for PA are presented in Table S1.

PA-R was defined as resistance to at least one of the following antimicrobial agents: antipseudomonal quinolone, cephalosporin, penicillin plus beta-lactamase inhibitors and carbapenem, aminoglycosides, monobactam and polymyxin. MDR-PA was defined as resistance to three or more antibiotic groups commonly active against the aforementioned antimicrobial categories. ${ }^{16}$ We categorized patients into "PA-R group" in case PA-R was isolated at the initial circumstances. Once an individual with bronchiectasis was infected with PA-R, they remained in the "PA-R" group for the remaining study period. 


\section{Survival analysis}

Survival was initially obtained from patients or their relatives by telephone or hospital records. For patients who could not be contacted by telephone, the admission record in our hospital or during regular follow-up was collected and the date of the last visit or follow-up was used as censored data. Survival duration was calculated as the duration between hospital admission and death or December 2016 (censored).

\section{Statistical analysis}

Descriptive statistics were presented as mean (SD) or median (interquartile range) or number (percentage) as appropriate. Chi-squared test, unpaired $t$-tests and Mann-Whitney $U$ test were used for between-group comparisons when appropriate. Multivariate logistic regression analysis was conducted to explore the factors of PA-R isolates, and the results were reported as odds ratios (ORs) and $95 \%$ CIs. Variables with $P<0.10$ in univariate analyses were entered into multivariate analyses as potential confounders using backward selection strategy. Multivariate Cox proportional hazard regression model was used to investigate the potential factors associated with survival, and variables with $P<0.10$ in bivariate analysis were included as independent variables in multiple Cox regression analysis. Survival curves were depicted with Kaplan-Meier plot and compared with log-rank test. Subgroup analysis was performed by only including patients with PA-R (MDR vs non-MDR). All comparisons were two sided, and $P$-values $\leq 0.05$ were considered statistically significant. All analyses were performed using SPSS 22.0 (IBM Corporation, Armonk, NY, USA).

\section{Results}

\section{Recruitment and overall clinical characteristics}

As shown in Figure 1, 747 patients were included in the final analysis after exclusion of 32 patients who mainly presented with traction bronchiectasis and 34 patients who did not have

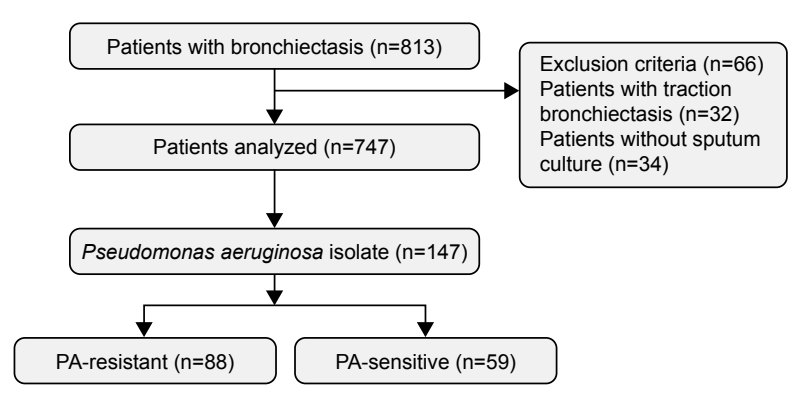

Figure I Flow chart of the selected population. Abbreviation: PA, Pseudomonas aeruginosa. undergone any bacterial culture. PA was isolated from the sputum in 147 (19.7\%) patients. Most patients with confirmed PA isolates had developed resistance to at least one category of antimicrobial agents (PA-R, $\mathrm{n}=88$ [59.9\%]), and $59(40.1 \%)$ had PA-sensitive (PA-S) isolate. Most patients with PA isolates were females ( $\mathrm{n}=82[55.8 \%])$, with a mean age of 59.7 years, mean $\mathrm{FEV}_{1}$ of $53.6 \%$ predicted and a mean HRCT score of 11.4. Pulmonary hypertension (35.4\%) was the most common comorbidity. Idiopathic and post-infectious bronchiectasis accounted for $31.3 \%$ and $29.9 \%$ of the underlying causes, respectively.

\section{Characteristics of bronchiectasis patients with and without PA-R}

Table 1 compares the demographic and clinical parameters between patients with and without PA-R isolates. There was no significant difference in age, sex, smoking status, lung function, comorbidities (except for pulmonary hypertension), prior use of medications and etiologies. However, patients with PA-R had lower BMI, more than two exacerbations and more than one hospitalization in the previous year, greater radiologic severity, more severe dyspnea and were more likely to have received systemic corticosteroids and antibiotics, when compared with those patients who had PA-S isolates. The antibiotic susceptibility pattern of PA isolates is shown in online supplement (Table S2).

\section{Risk factors for PA-R isolation}

For identifying the risk factors of PA-R isolates in bronchiectasis, we included the age, BMI, frequency of exacerbations and hospitalizations, modified Reiff HRCT score, mMRC score, $\mathrm{FEV}_{1} \%$ predicted, presence of pulmonary hypertension, use of systemic corticosteroids and antibiotic as independent variables in multivariate logistic regression. The model showed that systemic antibiotic treatment in the previous month $(\mathrm{OR}=6.18 ; 95 \% \mathrm{CI}, 2.22-17.19 ; P<0.001)$, more than two exacerbations in the previous year $(\mathrm{OR}=2.81$; 95\% CI, 1.02-7.77; $P=0.046$ ), higher mMRC scores $(\mathrm{OR}=1.93 ; 95 \% \mathrm{CI}, 1.34-2.78 ; P<0.001)$ and greater radiologic severity ( $\mathrm{OR}=1.15 ; 95 \% \mathrm{CI}, 1.04-1.28 ; P<0.001$ ) were independently associated with an increased risk of isolating PA-R strains (Table 2).

\section{Survival analysis}

Comparisons of survivors and nonsurvivors are shown in Table 3. Median follow-up lasted for 26 months (interquartile range: 6-59 months). All 147 (100\%) patients fully responded to the follow-up. Kaplan-Meier curves for patients 
Table I Demographic and clinical characteristics of bronchiectasis patients with and without PA-resistant in sputum

\begin{tabular}{|c|c|c|c|c|c|}
\hline Items & Parameters & $\begin{array}{l}\text { All patients } \\
(\mathrm{N}=147)\end{array}$ & $\begin{array}{l}\text { PA-R } \\
(n=88)\end{array}$ & $\begin{array}{l}\text { PA-S } \\
(n=59)\end{array}$ & $P$-value \\
\hline \multirow[t]{4}{*}{ Anthropometry } & Age, years & $59.7 \pm 18.2$ & $61.7 \pm 17.3$ & $56.7 \pm 19.1$ & 0.10 \\
\hline & Female & $82(55.8)$ & $46(52.3)$ & $36(61.0)$ & 0.30 \\
\hline & BMI $\left(\mathrm{kg} / \mathrm{m}^{2}\right)$ & $18.1 \pm 3.2$ & $17.4 \pm 2.4$ & $19.1 \pm 3.9$ & 0.001 \\
\hline & Nonsmokers & $107(72.8)$ & $61(69.3)$ & $46(78.0)$ & 0.25 \\
\hline \multirow[t]{4}{*}{ Disease-related parameters } & No of acute exacerbations within I year $>2$ & $95(64.6)$ & $69(78.4)$ & $26(44.1)$ & $<0.001$ \\
\hline & No of hospital admissions within I year $>$ I & $88(59.9)$ & $65(73.8)$ & $23(39.0)$ & $<0.001$ \\
\hline & Modified Reiff HRCT score & $11.4 \pm 4.7$ & $12.8 \pm 5.0$ & $9.4 \pm 3.4$ & $<\mathbf{0 . 0 0 1}$ \\
\hline & mMRC dyspnea score & $2.3 \pm 1.3$ & $2.8 \pm 1.2$ & $1.5 \pm 1.2$ & $<0.001$ \\
\hline \multirow[t]{2}{*}{ Lung function } & FVC pred \% & $65.3 \pm 19.4$ & $63.5 \pm 20.4$ & $68.0 \pm 17.6$ & 0.16 \\
\hline & $\mathrm{FEV}_{1}$ pred \% & $53.6 \pm 21.1$ & $51.4 \pm 22.7$ & $56.9 \pm 18.1$ & 0.12 \\
\hline \multirow[t]{5}{*}{ Comorbidity } & CHD & $18(12.2)$ & $12(13.6)$ & $6(10.2)$ & 0.53 \\
\hline & Hypertension & $30(20.4)$ & $20(22.7)$ & $10(16.9)$ & 0.39 \\
\hline & Stroke & $3(2.0)$ & $3(3.4)$ & $0(0)$ & 0.15 \\
\hline & Pulmonary hypertension & $52(35.4)$ & $43(48.9)$ & $9(15.3)$ & $<0.001$ \\
\hline & DM & $9(6.1)$ & $6(6.8)$ & $3(5.1)$ & 0.67 \\
\hline \multirow[t]{3}{*}{ Prior treatment } & Systemic corticosteroids (prior 3 months) & $81(55.1)$ & $59(67.0)$ & $22(37.3)$ & $<0.001$ \\
\hline & Antibiotics (prior 3 months) & $126(85.7)$ & $84(95.5)$ & $42(71.2)$ & $<0.001$ \\
\hline & Antibiotics (prior I month) & $95(64.6)$ & $64(72.7)$ & $31(52.5)$ & 0.01 \\
\hline \multirow[t]{4}{*}{ Current medications } & ICS & $71(48.3)$ & $42(47.7)$ & $29(49.1)$ & 0.87 \\
\hline & LABA & $68(46.3)$ & $40(45.5)$ & $28(47.5)$ & 0.81 \\
\hline & LAMA & $52(35.4)$ & $33(37.5)$ & $19(32.2)$ & 0.51 \\
\hline & Chronic macrolide treatment & $45(30.6)$ & $28(31.8)$ & $17(28.8)$ & 0.70 \\
\hline \multirow[t]{4}{*}{ Etiology } & Post-infectious & $44(29.9)$ & $25(28.4)$ & $19(32.2)$ & 0.62 \\
\hline & Immunodeficiency & $3(2.0)$ & $3(3.4)$ & $0(0)$ & 0.15 \\
\hline & Miscellaneous known causes & $54(36.7)$ & $31(35.2)$ & $23(39)$ & 0.64 \\
\hline & Idiopathic & $46(31.3)$ & $29(33.0)$ & $17(28.8)$ & 0.60 \\
\hline
\end{tabular}

Note: The significance of bold values $P<0.05$.

Abbreviations: BMI, body mass index; CHD, chronic heart disease; DM, diabetes mellitus; $\mathrm{FEV}_{1}$, forced expiratory volume in I second; FVC, forced vital capacity; HRCT, high-resolution computed tomography; ICS, inhaled corticosteroid; LABA, long-acting beta-agonist; LAMA, long-acting muscarinic antagonist; mMRC, modified Medical Research Council; PA, Pseudomonas aeruginosa; PA-R, PA-resistant; PA-S, PA-sensitive.

with PA-R (n=88; 24 deaths) and PA-S (n=59; 12 deaths) are shown in Figure 2. There was no significant difference between the two groups (log-rank test, $0.66 ; P=0.42$ ) in the survival rate. Table 4 shows the adjusted Cox regression analysis. Isolation of PA-R did not increase the risk of

Table 2 Factors associated with the presence of PA-resistant in patients with bronchiectasis in a logistic regression model

\begin{tabular}{lllll}
\hline Factors & B & Exp (B) & $\begin{array}{l}\mathbf{9 5 \%} \text { Cl } \\
\text { Exp (B) }\end{array}$ & P-value \\
\hline Antibiotics (prior I month) & 1.82 & 6.18 & $2.22-17.19$ & $<\mathbf{0 . 0 0 1}$ \\
$\begin{array}{l}\text { No of acute exacerbations } \\
\text { within I year }>2\end{array}$ & 1.03 & 2.81 & $1.02-7.77$ & $\mathbf{0 . 0 4 6}$ \\
mMRC dyspnea scores & 0.66 & 1.93 & $1.34-2.78$ & $<\mathbf{0 . 0 0 1}$ \\
Modified HRCT scores & 0.14 & 1.15 & $1.04-1.28$ & $<\mathbf{0 . 0 0 1}$ \\
Antibiotics (prior 3 months) & 1.28 & 3.60 & $0.90-14.44$ & 0.07 \\
\hline
\end{tabular}

Notes: Variables were adjusted for age, BMI, comorbid conditions, mMRC dyspnea score, acute exacerbations or hospitalizations in the past years, $\mathrm{FEV} \%$ of predicted, HRCT scores and prior treatment. The significance of bold values $P<0.05$.

Abbreviations: $B, B$ coefficient; BMI, body mass index; Exp (B), exponentiation of the $B$ coefficient; $F E V_{1}$, forced expiratory volume in I second; mMRC, modified Medical Research Council; HRCT, high-resolution computed tomography; PA, Pseudomonas aeruginosa. all-cause mortality in bronchiectasis (hazard ratio $=2.59$; 95\% CI, 0.83-8.11; $P=0.10)$. Aging, lower BMI and greater modified Reiff score had an independent adverse prognostic value in our fully adjusted model.

\section{Subgroup analysis}

To further investigate the effect of MDR resistance pattern, we performed a subgroup analysis by only including patients with PA-R ( $\mathrm{n}=88)$. Of these, 34 (38.6\%) had PA strains resistant to three or more antibiotics categories (MDR) and $54(61.4 \%)$ had PA strains resistant to less than three antibiotic categories (non-MDR).

There were no differences in demographic characteristics between MDR and non-MDR groups, except that the MDR group had a higher rate of more than one hospitalization in the previous year, higher mMRC score, greater lung function impairment and higher prevalence of pulmonary hypertension (Table S3). The potential risk factors for MDR-PA isolate included systemic antibiotic treatment in the previous month ( $\mathrm{OR}=4.28 ; 95 \% \mathrm{CI}, 1.43-12.79 ; P=0.009)$, more than two 
Table 3 Demographic and clinical characteristics of bronchiectasis patients according to survival status at the end of the study

\begin{tabular}{|c|c|c|c|c|}
\hline Items & Parameters & $\begin{array}{l}\text { Survivors } \\
(n=I I I)\end{array}$ & $\begin{array}{l}\text { Nonsurvivors } \\
(n=36)\end{array}$ & $P$-value \\
\hline \multirow[t]{4}{*}{ Anthropometry } & Age, years & $56.3 \pm 17.0$ & $72.4 \pm 11.8$ & $<0.001$ \\
\hline & Female & $66(59.5)$ & $16(44.4)$ & 0.12 \\
\hline & BMI $\left(\mathrm{kg} / \mathrm{m}^{2}\right)$ & $18.6 \pm 3.3$ & $16.5 \pm 1.9$ & $<0.001$ \\
\hline & Nonsmokers & $82(73.9)$ & $25(69.4)$ & 0.60 \\
\hline \multirow[t]{4}{*}{ Disease-related parameters } & No of acute exacerbations within I year $>2$ & $74(66.7)$ & $21(58.3)$ & 0.36 \\
\hline & No of hospital admissions within I year $>$ I & $68(61.3)$ & $20(55.6)$ & 0.54 \\
\hline & Modified Reiff HRCT score & $10.9 \pm 4.6$ & $13.0 \pm 4.6$ & 0.019 \\
\hline & mMRC dyspnea score & $2.2 \pm 1.3$ & $2.8 \pm 1.2$ & 0.019 \\
\hline \multirow[t]{2}{*}{ Lung function } & FVC pred \% & $68.2 \pm 20.2$ & $56.2 \pm 13.1$ & 0.001 \\
\hline & FEV , pred \% & $55.9 \pm 21.8$ & $46.4 \pm 17.2$ & 0.018 \\
\hline \multirow[t]{5}{*}{ Comorbid conditions } & $\mathrm{CHD}$ & $13(11.7)$ & $5(13.8)$ & 0.73 \\
\hline & Hypertension & $20(18.1)$ & $10(27.7)$ & 0.21 \\
\hline & Stroke & $2(1.8)$ & I (2.7) & 0.72 \\
\hline & Pulmonary hypertension & $34(30.6)$ & $18(50.0)$ & 0.035 \\
\hline & DM & $7(6.4)$ & $2(5.6)$ & 0.87 \\
\hline \multirow[t]{3}{*}{ Prior treatment } & Systemic corticosteroids (prior 3 months) & $54(48.6)$ & $27(75.0)$ & 0.006 \\
\hline & Antibiotics (prior 3 months) & $93(83.8)$ & 33 (9l.7) & 0.24 \\
\hline & Antibiotics (prior I month) & $68(61.3)$ & $27(75.0)$ & 0.13 \\
\hline \multirow[t]{4}{*}{ Current medications } & ICS & $51(45.9)$ & $20(55.6)$ & 0.32 \\
\hline & LABA & $48(43.2)$ & $20(55.6)$ & 0.20 \\
\hline & LAMA & $38(34.2)$ & 14 (38.9) & 0.61 \\
\hline & Chronic macrolide treatment & $35(31.5)$ & $10(27.8)$ & 0.67 \\
\hline \multirow[t]{4}{*}{ Etiology } & Post-infectious & $32(28.8)$ & $12(33.3)$ & 0.61 \\
\hline & Immunodeficiency & $3(2.7)$ & $0(0)$ & 0.19 \\
\hline & Miscellaneous known causes & $42(37.8)$ & $12(33.3)$ & 0.63 \\
\hline & Idiopathic & $34(30.6)$ & $12(33.3)$ & 0.76 \\
\hline PA & Isolate of PA-R & $64(57.7)$ & $24(66.7)$ & 0.34 \\
\hline
\end{tabular}

Note: The significance of bold values $P<0.05$.

Abbreviations: BMI, body-mass index; CHD, chronic heart disease; DM, diabetes mellitus; FEV, forced expiratory volume in I second; FVC, forced vital capacity; HRCT, high-resolution computed tomography; ICS, inhaled corticosteroid; LABA, long-acting beta-agonist; LAMA, long-acting muscarinic antagonist; mMRC, modified Medical Research Council; PA, Pseudomonas aeruginosa; PA-R, PA-resistant.

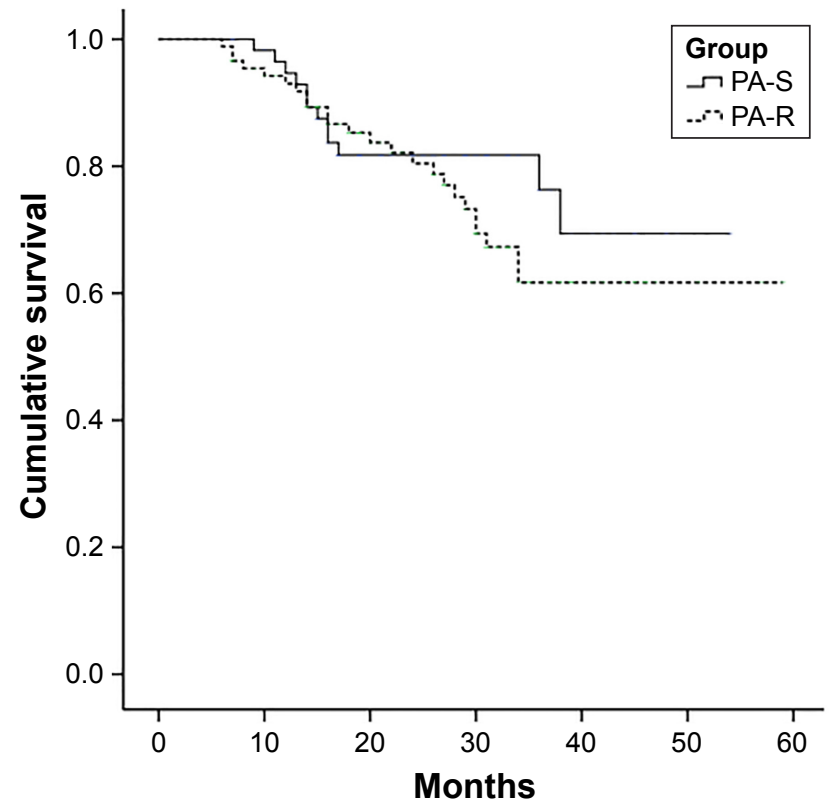

Figure 2 The Kaplan-Meier survival curves for bronchiectasis patients with PA-R isolate ( $\mathrm{n}=88 ; 24$ deaths) and with PA-S isolate ( $\mathrm{n}=59 ; 12$ deaths).

Note: Patients with PA-R isolate were not associated with increased mortality compared to those with PA-S isolate (log-rank test, 0.66; $P=0.42$ ).

Abbreviations: PA, Pseudomonas aeruginosa; PA-R, PA-resistant; PA-S, PA-sensitive. exacerbations in the previous year $(\mathrm{OR}=3.86 ; 95 \% \mathrm{CI}$, 1.07-13.92; $P=0.04)$, higher mMRC scores $(\mathrm{OR}=2.37$; 95\% CI, 1.26-4.47; $P=0.007)$ and lower $\mathrm{FEV}_{1} \%$ predicted (OR $=1.04 ; 95 \%$ CI, 1.01-1.06; $P=0.003)$, as shown in Table S4. However, there were no significant differences in all-cause mortality when stratified based on MDR status $(P=0.11)$.

Table 4 Variables associated with all-cause mortality in patients with bronchiectasis in a Cox proportional hazard regression model

\begin{tabular}{lllll}
\hline Factors & B & Exp (B) & $\begin{array}{l}\text { 95\% Cl } \\
\text { Exp (B) }\end{array}$ & P-value \\
\hline Age & 0.11 & 1.11 & $1.05-1.17$ & $<\mathbf{0 . 0 0 I}$ \\
BMI $\left(\mathrm{kg} / \mathrm{m}^{2}\right)$ & -0.60 & 0.55 & $0.39-0.76$ & $<\mathbf{0 . 0 0 I}$ \\
HRCT scores & 0.15 & 1.16 & $1.02-1.32$ & $\mathbf{0 . 0 2}$ \\
Isolates of PA-resistant & 0.95 & 2.59 & $0.83-8.11$ & 0.10 \\
\hline
\end{tabular}

Notes: The significance of bold values $P<0.05$. Variables were adjusted for age, $\mathrm{BMI}$, comorbid conditions, mMRC dyspnea score, $\mathrm{FEV}, \%$ of predicted, FVC\% of predicted and HRCT scores.

Abbreviations: B, B coefficient; BMI, body mass index; Exp (B), exponentiation of the B coefficient; $F E V_{1}$, forced expiratory volume in I second; FVC, forced vital capacity; HRCT, high-resolution computed tomography; mMRC, modified Medical Research Council; PA, Pseudomonas aeruginosa. 


\section{Discussion}

To date, limited data exist regarding the prevalence, risk factors and prognostic implications of PA-R isolates from bronchiectasis patients, especially in China. We found a high rate of PA-R isolates from hospitalized bronchiectasis patients. Systemic antibiotic exposures in the previous month, more than two exacerbations in the previous year, higher mMRC scores and greater radiologic severity were the major risk factors for isolating PA-R. However, isolation of PA-R did not increase all-cause mortality in bronchiectasis, suggesting that PA-R was a marker of more severe disease and more frequent or intensive antibiotic use, but was not associated with greater mortality.

PA infection in bronchiectasis presents a major challenge for clinicians, and has been associated with more severe disease and greater morbidity and mortality, emphasizing the importance of appropriate management of PA in clinical practice. ${ }^{7,17,18}$ Accordingly, susceptibility testing of PA to antimicrobial agents not only may guide the treatment for individual bronchiectasis patients, but is also valuable for identifying the prevalence of resistant strains within and between different treatment centers. ${ }^{17,19}$ In fact, susceptibility of PA isolated from patients with CF has been well described, ${ }^{19,20}$ whereas the profiles are scant in non-CF bronchiectasis. Our study showed that PA was isolated from nearly $20 \%$ of patients with bronchiectasis, with nearly two thirds presenting with PA-R isolates, which was concordant with McDonnell et al's report (resistance rate: 17/155, 11\%). ${ }^{12}$ As confirmed in our multivariable analysis, previous systemic antibiotic administration and repetitive exacerbations contributed considerably to PA-R isolation. In China, patients often self-medicate with oral antibiotics without consultation with physicians, which is possibly due to the lack of health education and awareness. ${ }^{21,22}$ Most patients with steady-state bronchiectasis had been prescribed systemic antibiotics when they attended respiratory clinics, especially for those with daily purulent sputum production. ${ }^{23}$ Additionally, when patients had an acute worsening of respiratory symptoms, they often received repeated and prolonged courses of antibiotics. ${ }^{23}$ Finally, antibiotic selective pressure and misuse may further predispose to the emergence of resistant bacteria, particularly PA-R that can be transmitted rapidly among patients who attend bronchiectasis clinics. Although there are no data about the risk factors of PA-R isolates in non-CF bronchiectasis, multiple studies in $\mathrm{CF}$ have shown that PA-R prevalence closely paralleled with the number of courses of antibiotic use..$^{8,11,24}$

During follow-up, 36(24.5\%) patients died, which mirrored the findings by Goeminne et $\mathrm{al}^{25}$ (median: 5.18 years, 20.4\%). PA infection has previously been linked to persistent and heightened lower airway inflammation which was associated with increased risk of exacerbation and death in bronchiectasis. ${ }^{2,7}$ Nonetheless, no studies had correlated the impact of PA-R infection with mortality of hospitalized bronchiectasis patients. Our study suggested that isolation of PA-R did not increase the risk of death compared with that in those from whom PA-S was isolated, suggesting the presence of PA-R was probably a marker of more severe disease and more frequent or intensive antibiotic administration, rather than being an independent contributor to poorer outcomes. Consistently, a recent study showed that MDR-PA was associated with greater doses of intravenous antibiotic administration, but not more adverse clinical outcomes in patients with $\mathrm{CF}{ }^{11}$ Montero et $\mathrm{al}^{26}$ reported that exacerbated COPD patients with PA-R isolates had higher mortality. Conversely, RodrigoTroyano et $\mathrm{al}^{10}$ demonstrated that the presence of PA-R was associated with lower mortality in COPD. These conflicting findings suggested that virulence of microorganisms might be determined by factors other than antibiotic resistance patterns. Alternatively, the comprehensive treatment for bronchiectasis exacerbation might have resulted in clinical improvement independent of the bactericidal effects of antibiotics. Of note, this study showed that PA-R isolate primarily represented a marker, which developed in sicker bronchiectasis patients who had frequent exacerbations and received repeated courses of antibiotics. Nevertheless, a larger prospective study with longer follow-up will be required to fully assess the effects of PA-R infection in bronchiectasis.

Noticeably, this study aimed to appreciate the clinical implications of bronchiectasis patients being identified initially as infected with PA-R. Therefore, patients were assigned to "PA-R group" once PA-resistant strains were initially isolated. Though bronchiectasis patients often have antibiotic-resistant strains isolated simultaneously or subsequently to the isolation of susceptible PA in clinical practice, it would be of significance for physicians to determine the impact on the prognosis after initial acquisition of PA-R isolates. Further studies are required to investigate the difference between patients who have intermittently positive PA-R cultures and those with persistently positive PA-R cultures. Additionally, 52 patients (35.4\%) were diagnosed as having PH (Table 1). The prevalence of PH has not been systematically investigated in adults with bronchiectasis. A study investigating 94 patients found that nearly one third had $\mathrm{PH}$ assessed with echocardiography. ${ }^{27}$ In our study, $48.9 \%$ of patients with PA-R had PH, as compared with $15.3 \%$ of patients with PA-S who had PH. The underlying mechanisms were unclear, but hypoxic vasoconstriction, mechanical stress of pulmonary hyperinflation, loss of pulmonary capillaries, 
spill over of airway inflammation and in situ thrombosis might have independently or collectively played a role. ${ }^{27,28}$ These changes might be more commonly seen in patients with PA-R, which warrant further investigations.

As with any observational study, several limitations merit considerations. First, the data were collected from a single tertiary hospital in China. Only adult bronchiectasis patients were included, which might have limited the generalizability of our findings to pediatric patients or those admitted to primary or secondary hospitals. Second, no control group with non-PA infection was included, despite that this study aimed to investigate the impact of antimicrobial resistance and its association with clinical outcomes. Nevertheless, many studies have shown that PA infections correlate with worse clinical outcomes, and therefore, recapitulation of these findings was not our main objective. Third, the types of PA strains, such as mucoid or non-mucoid, may be associated with antibiotic resistance, but this special pathogen characteristic was not evaluated herein. Fourth, we did not assess the association between individual resistance markers and prior antibiotic exposure. Fourth, the status of risk factors measured at baseline might change during follow-up (ie, weakened or strengthened) the association between PA-R infection and survival. Finally, some patients might have been misclassified. Patients were either in the "PA-R group" or "PA-S group" based on their initial sputum culture results. We did not assess multiple previous sputum cultures prior to their study entry. Therefore, an individual who had cultured PA-R previously might have been labeled erroneously as being susceptible at the time of study entry. Nevertheless, longitudinal assessment of microbiologic profiles in patients with bronchiectasis is urgently needed, which might help clinicians better appreciate the effects of PA-R infection on bronchiectasis.

\section{Conclusion}

In summary, infection with PA-R is common in hospitalized bronchiectasis patients and is independently associated with prior exposure to antibiotics, frequent exacerbations, higher mMRC scores and more severe radiologic appearances. However, PA-R isolation is not associated with poorer clinical outcomes. Future studies are needed to determine the molecular and genetic mechanisms underlying the increased virulence of PA regardless of antibiotic resistance patterns, which may help determine the optimal treatment regimens for bronchiectasis patients infected with PA-R.

\section{Acknowledgments}

Y-HG declared that he has received the National Natural Science Foundation No 81500006 and the Open Project of State Key Laboratory of Respiratory Disease No SKLRD2016OP013. W-JG declared that he has received the National Natural Science Foundation No 81400010, Pearl River S\&T Nova Program of Guangzhou No 201710010097, and Guangdong Province Universities and Colleges Pearl River Scholar Funded Scheme 2017.

\section{Author contributions}

Y-HG and W-JG drafted the manuscript; Y-NZ, R-CC and G-JZ were responsible for data collection; Y-HG performed statistical analyses; Y-HG, W-JG, R-CC and G-JZ contributed to study conception; R-CC and G-JZ provided critical review of the manuscript and approved the final submission. All authors contributed toward data analysis, drafting and revising the paper and agree to be accountable for all aspects of the work.

\section{Disclosure}

The authors report no conflicts of interest in this work.

\section{References}

1. Pasteur MC, Bilton D, Hill AT; British Thoracic Society Non-CF Bronchiectasis Guideline Group. British thoracic society guideline for non-CF bronchiectasis. Thorax. 2010;65(7):577.

2. Chalmers JD, Smith MP, McHugh BJ, Doherty C, Govan JR, Hill AT. Short- and long-term antibiotic treatment reduces airway and systemic inflammation in non-cystic fibrosis bronchiectasis. Am J Respir Crit Care Med. 2012;186(7):657-665.

3. Guan WJ, Gao YH, Xu G, et al. Sputum matrix metalloproteinase- 8 and -9 and tissue inhibitor of metalloproteinase- 1 in bronchiectasis: clinical correlates and prognostic implications. Respirology. 2015; 20(7):1073-1081.

4. Angrill J, Agusti C, de Celis R, et al. Bacterial colonisation in patients with bronchiectasis: microbiological pattern and risk factors. Thorax. 2002;57(1):15-19.

5. Guan WJ, Gao YH, Xu G, et al. Sputum bacteriology in steady-state bronchiectasis in Guangzhou, China. Int J Tubercul Lung Dis. 2015; 19(5):610-619.

6. Guan WJ, Gao YH, Xu G, et al. Effect of airway Pseudomonas aeruginosa isolation and infection on steady-state bronchiectasis in Guangzhou, China. J Thorac Dis. 2015;7(4):625-636.

7. Finch S, McDonnell MJ, Abo-Leyah H, Aliberti S, Chalmers JD. A comprehensive analysis of the impact of Pseudomonas aeruginosa colonization on prognosis in adult bronchiectasis. Ann Am Thorac Soc. 2015;12(11):1602-1611.

8. Merlo CA, Boyle MP, Diener-West M, Marshall BC, Goss CH, Lechtzin N. Incidence and risk factors for multiple antibiotic-resistant Pseudomonas aeruginosa in cystic fibrosis. Chest. 2007;132(2): 562-568.

9. Bradbury R, Champion A, Reid DW. Poor clinical outcomes associated with a multi-drug resistant clonal strain of Pseudomonas aeruginosa in the Tasmanian cystic fibrosis population. Respirology. 2008;13(6): 886-892.

10. Rodrigo-Troyano A, Suarez-Cuartin G, Peiro M, et al. Pseudomonas aeruginosa resistance patterns and clinical outcomes in hospitalized exacerbations of COPD. Respirology. 2016;21(7):1235-1242.

11. Smith DJ, Ramsay KA, Yerkovich ST, et al. Pseudomonas aeruginosa antibiotic resistance in Australian cystic fibrosis centres. Respirology. 2016;21(2):329-337.

12. McDonnell MJ, Jary HR, Perry A, et al. Non cystic fibrosis bronchiectasis: a longitudinal retrospective observational cohort study of Pseudomonas persistence and resistance. Respir Med. 2015;109(6):716-726. 
13. Pasteur MC, Helliwell SM, Houghton SJ, et al. An investigation into causative factors in patients with bronchiectasis. Am J Respir Crit Care Med. 2000;162(4 Pt 1):1277-1284.

14. Guan WJ, Gao YH, Xu G, et al. Aetiology of bronchiectasis in Guangzhou, southern China. Respirology. 2015;20(5):739-748.

15. Antimicrobial Susceptibility Testing. Performance Standards for Testing: Twenty-First Informational Supplement M100-S21. Wayne, PA, USA: Clinical and Laboratory Standards Institute; 2011.

16. Magiorakos AP, Srinivasan A, Carey RB, et al. Multidrug-resistant, extensively drug-resistant and pandrug-resistant bacteria: an international expert proposal for interim standard definitions for acquired resistance. Clin Microbiol Infect. 2012;18(3):268-281.

17. Wilson R, Aksamit T, Aliberti S, et al. Challenges in managing Pseudomonas aeruginosa in non-cystic fibrosis bronchiectasis. Respir Med. 2016;117:179-189.

18. Guan WJ, Gao YH, Xu G, et al. Effect of airway Pseudomonas aeruginosa isolation and infection on steady-state bronchiectasis in Guangzhou, China. J Thorac Dis. 2015;7(4):625-636.

19. Ren CL, Konstan MW, Yegin A, et al; Scientific Advisory Group, Investigators, and Coordinators of the Epidemiologic Study of Cystic Fibrosis. Multiple antibiotic-resistant Pseudomonas aeruginosa and lung function decline in patients with cystic fibrosis. J Cyst Fibros. 2012; 11(4):293-299.

20. Rao P, McCaughan J, McCalmont M, et al. Comparison of antibiotic susceptibility patterns in Pseudomonas aeruginosa isolated from adult patients with cystic fibrosis (CF) with invasive Pseudomonas aeruginosa from non-CF patients. J Cyst Fibrosis. 2012;11(4):349-352.
21. Quan Cheng K, Jian-Guo W, Xiang-Hua L, Zhen-Zhen L. Inappropriate use of antibiotics in children in China. Lancet. 2016;387(10025): 1273-1274.

22. Xiao Y, Li L. Legislation of clinical antibiotic use in China. Lancet Infect Dis. 2013;13(3):189-191.

23. Lin JL, Xu JF, Qu JM. Bronchiectasis in China. Ann Am Thorac Soc. 2016;13(5):609-616.

24. Pitt TL, Sparrow M, Warner M, Stefanidou M. Survey of resistance of Pseudomonas aeruginosa from UK patients with cystic fibrosis to six commonly prescribed antimicrobial agents. Thorax. 2003;58(9): 794-796.

25. Goeminne PC, Nawrot TS, Ruttens D, Seys S, Dupont LJ. Mortality in non-cystic fibrosis bronchiectasis: a prospective cohort analysis. Respir Med. 2014;108(2):287-296.

26. Montero M, Dominguez M, Orozco-Levi M, Salvado M, Knobel H. Mortality of COPD patients infected with multi-resistant Pseudomonas aeruginosa: a case and control study. Infection. 2009;37(1):16-19.

27. Alzeer AH, AI-Mobeirek AF, Al-Otair HA, Elzamzamy UA, Joherjy IA, Shaffi AS. Right and left ventricular function and pulmonary artery pressure in patients with bronchiectasis. Chest. 2008;133(2):468-473.

28. Devaraj A, Wells AU, Meister MG, Loebinger MR, Wilson R, Hansell DM. Pulmonary hypertension in patients with bronchiectasis: prognostic significance of CT signs. AJR Am J Roentgenol. 2011;196(6): 1300-1304. 


\section{Supplementary materials}

Table SI MIC interpretive standards of the disk diffusion methodologies for PA according to the CLSI

\begin{tabular}{|c|c|c|c|}
\hline \multirow[t]{2}{*}{ Antibiotic } & \multicolumn{3}{|c|}{ MIC interpretive standard $(\mu \mathrm{g} / \mathrm{mL})$} \\
\hline & $\mathbf{S}$ & $\mathbf{I}$ & $\mathbf{R}$ \\
\hline Gentamicin & $\leq 4$ & 8 & $\geq 16$ \\
\hline Tobramycin & $\leq 4$ & 8 & $\geq 16$ \\
\hline Amikacin & $\leq 16$ & 32 & $\geq 64$ \\
\hline Ceftazidime & $\leq 8$ & 16 & $\geq 32$ \\
\hline Cefepime & $\leq 8$ & 16 & $\geq 32$ \\
\hline Ticarcillin-clavulanate & $\leq 64 / 2$ & - & $\geq 128 / 2$ \\
\hline Piperacillin-tazobactam & $\leq 64 / 4$ & - & $\geq 128 / 4$ \\
\hline Aztreonam & $\leq 8$ & 16 & $\geq 32$ \\
\hline Meropenem & $\leq 4$ & 8 & $\geq 16$ \\
\hline Imipenem & $\leq 4$ & 8 & $\geq 16$ \\
\hline Ciprofloxacin & $\leq 1$ & 2 & $\geq 4$ \\
\hline Levofloxacin & $\leq 2$ & 4 & $\geq 8$ \\
\hline Colistin sulfate & $\leq 2$ & 4 & $\geq 8$ \\
\hline
\end{tabular}

Abbreviations: CLSI, Clinical and Laboratory Standards Institute; I, intermediate; MIC, minimal inhibitory concentration; PA, Pseudomonas aeruginosa; R, resistant; S, sensitive.

Table S2 Antibiotic resistance profile of I 47 Pseudomonas aeruginosa isolates

\begin{tabular}{ll}
\hline Antibiotic & Number of susceptible strains (\%) \\
\hline Ciprofloxacin & $114(77.6)$ \\
Piperacillin-tazobactam & $12 I(82.3)$ \\
Ceftazidime & $121(82.3)$ \\
Imipenem & $95(64.6)$ \\
Gentamicin & $13 \mid(89.1)$ \\
Amikacin & $14 \mid(95.9)$ \\
\hline
\end{tabular}


Table S3 Baseline and clinical characteristics of bronchiectasis patients with PA-R in non-MDR and MDR groups in sputum

\begin{tabular}{|c|c|c|c|c|}
\hline Items & Parameters & $\begin{array}{l}\text { Non-MDR } \\
(n=54)\end{array}$ & $\begin{array}{l}\text { MDR } \\
(n=34)\end{array}$ & $P$-value \\
\hline \multirow[t]{4}{*}{ Anthropometry } & Age, years & $62.7(13.7)$ & $60.2(22.1)$ & 0.52 \\
\hline & Female & $31(57.4)$ & $15(44.1)$ & 0.22 \\
\hline & BMI $\left(\mathrm{kg} / \mathrm{m}^{2}\right)$ & $17.4(2.9)$ & $17.6(1.2)$ & 0.74 \\
\hline & Nonsmokers & $40(74.1)$ & $21(61.8)$ & 0.22 \\
\hline \multirow[t]{4}{*}{ Disease-related parameters } & No of acute exacerbations within I year $>2$ & $39(72.2)$ & $30(88.2)$ & 0.08 \\
\hline & No of hospital admissions within I year $>$ I & $36(66.7)$ & $29(85.3)$ & 0.05 \\
\hline & Modified Reiff HRCT score & $12.3(5.1)$ & I $3.6(4.8)$ & 0.25 \\
\hline & mMRC dyspnea score & $2.5(1.3)$ & $3.3(0.5)$ & 0.003 \\
\hline \multirow[t]{2}{*}{ Lung function } & FVC pred \% & $69.4(21.0)$ & $54.1(15.5)$ & $<0.001$ \\
\hline & $\mathrm{FEV}$, pred \% & $58.6(23.6)$ & $40.0(15.6)$ & $<0.001$ \\
\hline \multirow[t]{5}{*}{ Comorbid conditions } & $\mathrm{CHD}$ & $9(16.7)$ & $3(8.8)$ & 0.30 \\
\hline & Hypertension & $12(22.2)$ & $8(23.5)$ & 0.89 \\
\hline & Stroke & $0(0)$ & $3(8.8)$ & 0.16 \\
\hline & Pulmonary hypertension & $20(37.0)$ & $23(67.6)$ & 0.01 \\
\hline & DM & $6(11.1)$ & $3(8.8)$ & 0.73 \\
\hline \multirow[t]{3}{*}{ Prior treatment } & Systemic corticosteroids (prior 3 months) & $33(61.1)$ & $26(76.5)$ & 0.14 \\
\hline & Antibiotics (prior 3 months) & $5 \mathrm{I}(94.4)$ & $33(97.1)$ & 0.57 \\
\hline & Antibiotics (prior I month) & $36(66.7)$ & $28(82.4)$ & 0.11 \\
\hline \multirow[t]{4}{*}{ Current medications } & ICS & $26(48.1)$ & $16(47.1)$ & 0.92 \\
\hline & LABA & $24(44.4)$ & $16(47.1)$ & 0.81 \\
\hline & LAMA & $19(35.2)$ & $14(4 \mid .2)$ & 0.57 \\
\hline & Chronic macrolide treatment & $13(24.1)$ & $15(44.1)$ & 0.18 \\
\hline \multirow[t]{4}{*}{ Etiology } & Post-infectious & $15(27.7)$ & $10(29.4)$ & 0.87 \\
\hline & Immunodeficiency & I (I.9) & $2(5.9)$ & 0.31 \\
\hline & Miscellaneous known causes & $18(33.3)$ & $13(38.2)$ & 0.64 \\
\hline & Idiopathic & $20(37.0)$ & $10(29.4)$ & 0.46 \\
\hline
\end{tabular}

Abbreviations: BMI, body-mass index; CHD, chronic heart disease; DM, diabetes mellitus; FEV , forced expiratory volume in I second; FVC, forced vital capacity; HRCT, high-resolution computed tomography; ICS, inhaled corticosteroid; LABA, long-acting beta-agonist; LAMA, long-acting muscarinic antagonist; MDR, multidrug resistant; mMRC, modified Medical Research Council; PA, Pseudomonas aeruginosa; PA-R, PA-resistant.

Table S4 Factors associated with the presence of MDR-PA in patients with bronchiectasis in a logistic regression model

\begin{tabular}{lllll}
\hline Factors & B & Exp (B) & 95\% CI Exp (B) & P-value \\
\hline Antibiotics (prior I month) & 1.45 & 4.28 & $1.43-12.79$ & $\mathbf{0 . 0 0 9}$ \\
No of acute exacerbations within I year $>2$ & 1.35 & 3.86 & $1.07-13.92$ & $\mathbf{0 . 0 4}$ \\
mMRC dyspnea scores & 0.86 & 2.37 & $1.26-4.47$ & $\mathbf{0 . 0 0 7}$ \\
Lower FEV , pred \% & 0.04 & 1.04 & $1.01-1.06$ & $\mathbf{0 . 0 0 3}$ \\
\hline
\end{tabular}

Notes: The significance of bold values $P<0.05$. Variables were adjusted for age, BMI, comorbid conditions, mMRC dyspnea, acute exacerbations or hospitalizations in the past years, FEV \% of predicted, FVC\% of predicted.

Abbreviations: B, B coefficient; BMI, body mass index; $\operatorname{Exp}(B)$, exponentiation of the B coefficient; FEV , forced expiratory volume in I second; FVC, forced vital capacity; MDR, multidrug resistant; mMRC, modified Medical Research Council; PA, Pseudomonas aeruginosa.

\section{Publish your work in this journal}

The International Journal of COPD is an international, peer-reviewed journal of therapeutics and pharmacology focusing on concise rapid reporting of clinical studies and reviews in COPD. Special focus is given to the pathophysiological processes underlying the disease, intervention programs, patient focused education, and self management protocols.

\section{Dovepress}

This journal is indexed on PubMed Central, MedLine and CAS. The manuscript management system is completely online and includes a very quick and fair peer-review system, which is all easy to use. Visit $\mathrm{http}: / /$ www.dovepress.com/testimonials.php to read real quotes from published authors. 